\title{
SEZIONE RECENSIONI
}

di Gennaro Iasevoli (iasevoli@lumsa.it; gennaro.iasevoli@gmail.com)

In questo spazio presentiamo alcuni fra i più recenti e autorevoli testi su tematiche incentrate sulle attività e sugli strumenti di marketing, pubblicati sia a livello nazionale che internazionale. Per eventuali segnalazioni contattare Gennaro Iasevoli via e-mail.

\begin{abstract}
Abirascid Emil
L'innovazione che non ti aspetti. Contesti e visioni per l'impresa

FrancoAngeli, 2015

Il volume parte da alcuni principi: fare impresa è impegnativo, farla innovativa ancora di più, farla in Italia è una sfida. Alcuni interlocutori dell'imprenditoria innovativa hanno voluto condividere le loro storie in questo volume, storie di impresa ma anche idee e proposte, di modelli di business e di visione verso un futuro possibile. Storie che si innestano nello scenario e nel contesto di un Paese che ancora con difficoltà favorisce le forme di imprenditoria fortemente innovativa.
\end{abstract}

\section{Barbarossa Camilla}

Casi di marketing

FrancoAngeli, 2015

L'undicesimo testo della collana "Giorgio Eminente" del MUMM, affronta case histories inedite e l'analisi di concrete esperienze d'impresa. Questo volume della collana presenta le storie di: Telecom Italia, Criteo, Consul-marketing, Consorzio per la Tutela dell'Asti docg e i contributi dei relatori intervenuti al convegno "Competitività e Relazioni di Filiera" tra cui Centromarca, Conad, Peroni-SAB Miller, Procter \& Gamble.

\section{Boccardelli Paolo \\ Corporate strategy \\ Hoepli, 2015}

Il volume affronta il tema della strategia di impresa, argomento di grande rilevanza in questa fase del ciclo economico in cui tutte le aziende sono chiamate a ottimizzare la propria struttura strategica e a rinnovare il portafoglio delle opzioni di crescita. Attraverso una prospettiva che si focalizza su quattro pilastri della strategia - industry, resources, managerial competencies e institutions - il volume riserva ampio spazio all'analisi dell'implementazione delle strategie, al confronto con la realtà nazionale e internazionale e fornisce un approccio interdisciplinare che consente di analizzare le strategie con tre diverse visioni: quella progettuale strategica, quella organizzativa e quella finanziaria.

\section{Cherubini Sergio}

Marketing e management dello sport FrancoAngeli, 2015

Il volume propone con un approccio al tempo stesso rigoroso dal punto di vista scientifico e pratico da quello applicativo, molti casi ed esperienze relative a tutti i settori dello sport (dalla pratica allo spettacolo, dal professionismo al

Mercati e Competitività n. 3, 2015 
dilettantismo, dalla produzione ai servizi, dall'internazionalità al localismo, eccetera), opportunamente declinati nel marketing alle persone (B2C), alle aziende (B2B), alla pubblica amministrazione e alle istituzioni (B2P), nell'ambito di una convergenza sportiva sempre più integrata e sinergica. Questa edizione aggiornata include un approfondimento su alcune tematiche tra cui: le sponsorizzazioni, i diritti d'immagine e trasmissione, il merchandising e licensing, le scuole sportive, il brand e l'event management, il comarketing e l'heritage marketing. Il testo si compone di tre sezioni: il consumer marketing sportivo ovvero marketing alle persone, il business marketing sportivo ovvero marketing alle aziende, istituzioni, pubblica amministrazione, gli aspetti manageriali del marketing sportivo.

\section{Semoli Alessio \\ Marketing automation \\ Hopeli, 2015}

La Marketing Automation consente di automatizzare i processi legati a qualsiasi business online e di utilizzare strategie di web marketing e piattaforme che dialogano tra loro in automatico, incrementando così il numero delle conversioni e delle vendite generate dal proprio sito. Questo libro illustra la Marketing Automation per tutte le azioni di marketing e analizza tutti gli strumenti attualmente per semplificare ogni attività online. Fra gli argomenti trattati: Lead Generation: gestire un contatto attraverso attività di "nurturing" e "scoring"; Advertising Automation: dal Programmatic buying al SEM e al Social advertising automatizzato; Inbound Marketing: attrarre clienti attraverso strategie di Content marketing; Workflow automatizzati: mettere in campo strategie di Digital marketing.

\section{Martini Umberto}

Management dei sistemi territoriali. Gestione e marketing delle destinazioni turistiche

Giappichelli, 2015

Il testo analizza, all'interno di una prospettiva economico-manageriale, i processi di formulazione delle strategie di offerta turistica del territorio, con enfasi particolare alla definizione delle strutture organizzative necessarie per l'azione di marketing. Lo sviluppo in chiave turistica è affrontato ponendo al centro il sistema territoriale e l'attività delle imprese operanti al suo interno. Un ruolo rilevante, è giocato dagli organi amministrativi locali, i quali hanno la responsabilità di promuovere e sostenere lo sviluppo locale anche attraverso la realizzazione di strutture ed infrastrutture, e la creazione di organizzazioni per la gestione dei processi di marketing.

\section{Mazzei Alessandra}

Dialogare con gli stakeholder. Ascolto e sensibilità interculturale per le relazioni pubbliche e la comunicazione d'impresa

FrancoAngeli, 2015

La comunicazione d'impresa e le relazioni pubbliche hanno assunto oggi il significato di conversazione con gli stakeholder. Il volume approfondisce tre aspetti per illustrare il cambiamento nella comunicazione: teorie dialogiche, ascolto e interculturalità. Alcune teorie delle relazioni pubbliche evidenziano 
la natura interattiva e dialogica della comunicazione. In questo processo di dialogo ci sono due pratiche di particolare rilevanza: l'ascolto e la comunicazione interculturale. L'ascolto è il processo di apertura verso gli interlocutori per comprenderne aspettative e atteggiamenti. La comunicazione interculturale è basata sulla consapevolezza degli eventuali filtri culturali, soprattutto quando il pubblico ha un background differente.

Osterwalder Alexander, Pigneur Yves, Bernarda Gregory, Smith Alan

Value Proposition Design. Come creare prodotti e servizi che $i$ clienti desiderano

Edizioni LSWR, 2015

Applicando la stessa impostazione del canvas sui modelli di business, questo volume mostra come declinare il "Value Proposition Canvas", per progettare, collaudare e immettere sul mercato prodotti e servizi potenzialmente di successo. Esercizi, illustrazioni e strumenti pratici consentono di migliorare l'idea di business legata al prodotto. Il libro, inoltre, dà accesso esclusivo a un insieme di materiali di supporto quali esercizi interattivi, test di autovalutazione e casi di studio.

\section{Pencarelli Tonino (a cura di)}

Comunicare le destinazioni balneari. Il ruolo delle bandiere blu in Italia FrancoAngeli, 2015

Il volume affronta un tema cruciale per la competitività delle destinazioni balneari: come migliorare la comunicazione turistica combinando i tradizionali processi di Destination branding (tra cui quelli legati al brand della "Bandiera Blu"). Il testo colloca il ricorso alla certificazione ambientale nell'ambito dei processi di governo strategico e di Destination branding dei territori. Vengono descritte le dinamiche del turismo balneare in Italia, mettendo in luce sintomi di declino e l'esigenza di accrescere l'attenzione alla sostenibilità ambientale per rilanciare le località costiere. L'originalità del volume consiste anche nell'analisi dei risultati di un'indagine empirica riferita a 131 Comuni insigniti del brand Bandiera Blu, che mostra il grado di integrazione delle certificazioni con le politiche di branding.

\section{Siano Alfonso, Vollero Agostino, Si- glioccolo Mario}

Corporate communication management. Accrescere la reputazione per attrarre risorse

Giappichelli, 2015

La comunicazione aziendale è al centro di profondi cambiamenti: la crescente centralità attribuita all'obiettivo di reputazione; il ruolo proattivo del comunicatore nei processi decisionali; la diffusione delle pratiche di stakeholder engagement; le incessanti innovazioni dei media digitali; l'affermazione del consumer empowerment e degli usergenerated media and content. In rapporto a tali fattori di cambiamento, il libro propone un modello di corporate communication fondato su una prospettiva manageriale che garantisce l'assunzione di corrette decisioni e lo svolgimento di efficaci ed efficienti attività di comunicazione, a livello sia strategico che operativo. 